\title{
A non-pulsating neutron star in the supernova remnant HESS J1731-347/G353.6-0.7 with a carbon atmosphere
}

\author{
D. Klochkov ${ }^{1}$, G. Pühlhofer ${ }^{1}$, V. Suleimanov ${ }^{1,2}$, S. Simon ${ }^{1}$, K. Werner ${ }^{1}$, and A. Santangelo ${ }^{1}$ \\ ${ }^{1}$ Institut für Astronomie und Astrophysik, Universität Tübingen (IAAT), Sand 1, 72076 Tübingen, Germany \\ e-mail: klochkov@astro.uni-tuebingen.de \\ 2 Kazan (Volga region) Federal University, Kremlevskaya 18, 420008 Kazan, Russia
}

Received 19 April 2013 / Accepted 8 June 2013

\begin{abstract}
Context. The central compact object (CCO) candidate in the center of the supernova remnant shell HESS J1731-347/G353.6-0.7 shows no pulsations and exhibits a blackbody-like X-ray spectrum. If the absence of pulsations is interpreted as evidence for the emitting surface area being the entire neutron star surface, the assumption of the measured flux being due to a blackbody emission translates into a source distance that is inconsistent with current estimates of the remnant's distance.

Aims. With the best available observational data, we extended the pulse period search down to a sub-millisecond time scale and used a carbon atmosphere model to describe the X-ray spectrum of the $\mathrm{CCO}$ and to estimate geometrical parameters of the neutron star. Methods. To search for pulsations we used data of an observation of the source with XMM-Newton performed in timing mode. For the spectral analysis, we used earlier XMM-Newton observations performed in imaging mode, which permits a more accurate treatment of the background. The carbon atmosphere models used to fit the CCO spectrum are computed assuming hydrostatic and radiative equilibria and take into account pressure ionization and the presence of spectral lines.

Results. Our timing analysis did not reveal any pulsations with a pulsed fraction above $\sim 8 \%$ down to $0.2 \mathrm{~ms}$. This finding further supports the hypothesis that the emitting surface area is the entire neutron star surface. The carbon atmosphere model provides a good fit to the CCO spectrum and leads to a normalization consistent with the available distance estimates of the remnant. The derived constraints on the mass and radius of the source are consistent with reasonable values of the neutron star mass and radius. After the CCO in Cas A, the CCO in HESS J1731-347/G353.6-0.7 is the second object of this class for which a carbon atmosphere model provides a consistent description of X-ray emission.
\end{abstract}

Key words. stars: neutron - stars: atmospheres - ISM: supernova remnants

\section{Introduction}

The observed population of isolated neutron stars in the Galaxy is dominated by radio pulsars $(\sim 2000)$. The class of radio-quiet $\mathrm{X}$-ray emitting isolated neutron stars is much less numerous, only several tens. Members of this class appear in four different flavors: anomalous X-ray pulsars (AXP), soft gammaray repeaters (SGR), central compact objects (CCO), and X-ray dim isolated neutron stars (XDINS). The sources from the former three classes are in many cases associated with supernova remnants (SNR) and, thus, are believed to be relatively young $\left(\sim 10^{3}-10^{4} \mathrm{yr}\right)$. Central compact objects are defined through their locations near the geometrical center of young SNRs, their steady flux, predominantly thermal X-ray emission $(k T \sim$ $0.2-0.5 \mathrm{keV}$ ), and the lack of associated pulsar wind nebula (PWN) emission (e.g., Pavlov et al. 2004; Halpern \& Gotthelf 2010b, for recent reviews). Currently, less than ten such objects are known. Pulse periods have been measured for three of them and range from 0.1 to $0.4 \mathrm{~s}$. The spin-down rates or the corresponding upper limits for these three pulsars lead to relatively small estimated magnetic field strengths: $\$ 10^{10} \div 10^{11} \mathrm{G}$. These estimates led Halpern \& Gotthelf (2010b) to name these objects anti-magnetars. The characteristic age of these pulsars derived from the $P / \dot{P}$ ratio is much larger than the associated SNR age, which means that the pulsars were born with spin periods close to their present ones, assuming that the magnetic field stayed constant (see, however, Ho 2011; Viganò \& Pons 2012).
The recently discovered CCO candidate XMMUJ173203.3344518 (Acero et al. 2009; Tian et al. 2010) is a point-like source located close to the geometrical center of the shelltype supernova remnant HESS J1731-347 or G 353.6-0.7. The SNR itself is quite unusual as it was first discovered as a strong $\mathrm{TeV}$ emitter with H.E.S.S. (Aharonian et al. 2008), and only subsequently was the corresponding weak radio SNR shell identified (Tian et al. 2008). Thus, the remnant belongs to a relatively rare class of $\mathrm{TeV}$-emitting SNRs with only six other TeV-emitting SNRs known so far (e.g., Aharonian 2013). The source XMMUJ173203.3-344518 was discovered during $20 \mathrm{ks}$ follow-up observations of the SNR with XMM-Newton in 2007 and was in the FOV during subsequent Suzaku (Bamba et al. 2012) and Chandra observations of the remnant. The X-ray spectrum of the source can be well described by an absorbed blackbody model with $k T \simeq 0.5 \mathrm{keV}$ and an absorption column density of $n_{\mathrm{H}} \simeq 1.5 \times 10^{22} \mathrm{~cm}^{-2}$ (Acero et al. 2009; Halpern \& Gotthelf 2010c; Bamba et al. 2012). A possible deviation from a pure blackbody model has been discussed by Tian et al. (2010) and Halpern \& Gotthelf (2010c) and was modeled through an additional higher temperature blackbody or hard power-law component. However, contamination of the point-source spectrum by the surrounding SNR could be responsible for one or several additional components as demonstrated in Bamba et al. (2012), for example.

So far, no long-term X-ray variability has been detected for XMMUJ173203.3-344518 (Halpern \& Gotthelf 2010c). 
Tian et al. (2010) claimed that the additional hard spectral component was different in the 2007 Suzaku and XMM-Newton observations of the source, but given the uncertainties of the spectral extraction for this hard component, no strong conclusions can be drawn.

Measurements of X-ray pulsations are crucial for the characterization of a CCO. So far, observations have not revealed any hint of pulsations in XMMUJ173203.3-344518 down to $10 \mathrm{~ms}$ with an upper limit for the amplitude of a sinusoidal signal of $\sim 10 \%$ (Halpern \& Gotthelf 2010a). The absence of pulsations or a very low pulsed fraction indicate that the observed thermal radiation of the $\mathrm{CCO}$ is emitted by nearly the entire neutron star surface. There is also a chance that the spin axis of the pulsar points under a small angle towards Earth or that the emitting regions are located at the rotation poles of the star, which would suppress pulsations. For this paper, we adopt the hypothesis of a near-complete neutron star surface emission region.

If thus the entire neutron star surface is the source of emission, an interpretation of the spectrum as pure blackbody radiation would place the star at a distance of $30 \mathrm{kpc}$ (for a stellar radius of $10 \mathrm{~km}$ ). Such a large distance would imply an exceptionally high $\mathrm{TeV}$ luminosity of the remnant shell compared to other known TeV-emitting SNRs. Together with the SNR's lower distance limit of $\sim 3.2 \mathrm{kpc}$ and using the same luminosity argument, Abramowski et al. (2011) argued for a likely localization of HESS J1731-347 either in the Scutum-Crux arm or in the Norma-Cygnus arm, with the corresponding spiral arm distances of $\sim 3 \mathrm{kpc}$ or $\sim 4.5 \mathrm{kpc}$ following the model by Hou et al. (2009) for a Galactic center distance of $8.5 \mathrm{kpc}$.

A similar discrepancy between the distance estimates to the SNR shell and to the associated neutron star has been reported for another object, the non-pulsating CCO in the Cas A supernova remnant (see, e.g., Pavlov \& Luna 2009, and references therein), for which the distance has been measured very reliably (e.g., Reed et al. 1995). For Cas A, a plausible solution of the discrepancy has been presented by Ho \& Heinke (2009), who fitted the observed spectra of the CCO using a neutron star carbon atmosphere model. Their analysis brought the emitting area in agreement with the total surface area of a canonical neutron star.

In the present work, we also use a carbon atmosphere spectral model to reconcile the distance estimates of the SNR HESS J1731-347 with the emitting area of the CCO in its center which we have assumed to be the entire surface of the neutron star. Before doing so, we used the recent timing observations of the source with XMM-Newton to confirm the absence of pulsations down to $\sim 0.2 \mathrm{~ms}$ and the absence of any long-term flux variations, as well.

The paper is organized as follows. The observation history is provided in Sect. 2, where the long-term stability of the source's flux is discussed. The timing analysis, including the search for pulsations, is described in Sect. 3. The spectral analysis is described in Sect. 4. The details of the carbon atmosphere model and the procedure we used to fit it to the spectrum are described in Sect. 5 where we also discuss the constraints on the mass and radius of the neutron star. The results are summarized in Sect. 6.

\section{Long-term stability of XMMUJ173203.3-344518}

The CCO in HESS J1731-347 has been observed several times with the XMM-Newton, Chandra, Suzaku, and Swift orbital observatories. Table 1 provides a short summary of the observations. Most of them were aimed at the diffuse emission of the
Table 1. Summary of observations of XMMUJ173203.3-344518.

\begin{tabular}{lccc}
\hline \hline Date & Satellite & $\begin{array}{c}\text { Exposure } \\
{[\mathrm{ks}]}\end{array}$ & Time res. \\
\hline 2007 Feb. 23 & Suzaku & 41 & $8 \mathrm{~s}$ \\
2007 Mar. 21 & XMM-Newton & 25 & $70 \mathrm{~ms}(\mathrm{PN})$ \\
2008 Apr. 28 & Chandra & 30 & $3.2 \mathrm{~s}$ \\
2009 Feb. 4 & Swift & 1.4 & $2.5 \mathrm{~s}$ \\
2009 Mar. 9 & Swift & 1.4 & $2.5 \mathrm{~s}$ \\
2010 May 18 & Chandra & 40 & $2.85 \mathrm{~ms}$ \\
2012 Mar. 2 & XMM-Newton & 24 & $0.03 \mathrm{~ms}(\mathrm{PN})$ \\
\hline
\end{tabular}

supernova remnant shell and, therefore, were performed in imaging modes with limited time resolution. The last two observations in the table were, however, targeted at the CCO and performed in timing modes providing sufficient time resolution to search for pulsations down to a millisecond time scale. There were several short Swift observations of the source with exposures below $1 \mathrm{ks}$ each, which are not listed in the table.

The source XMMUJ173203.3-344518 is unresolved down to Chandra resolution. Halpern \& Gotthelf (2010c) reported an $\mathrm{X}$-ray nebulosity around the point source out to $\sim 50^{\prime \prime}$, the characteristics of which are compatible with a scattering halo. From these observations and the others as well there is no evidence for a pulsar wind nebula around the source, in agreement with the CCO hypothesis for XMMUJ173203.3-344518.

To check for any long-term variations of the source, we compiled the integrated point source flux measurements derived from the XMM-Newton, Chandra, and Suzaku observations in Fig. 1. Fluxes from the 2007 and 2008 data were derived from a single blackbody fit. Halpern \& Gotthelf (2010c) already noticed that the source fluxes from these data (also including the 2009 Swift measurements) are consistent within $20 \%$, and excluded magnetar-like activity. To the light curve we added the results from the two timing-mode measurements, the Chandra 2010 flux point derived by Halpern \& Gotthelf (2010a) with a twoblackbody fit as favored by these authors, and the XMM-Newton 2012 flux point as derived from a single blackbody fit. The new flux measurement, $F_{0.5-10 \mathrm{keV}}=2.37(4) \times 10^{-12} \mathrm{erg} \mathrm{cm}^{-2} \mathrm{~s}^{-1}(\mathrm{ab}-$ sorbed), indicates a flux that is a few sigma lower than that measured with XMM-Newton in 2007 and with the other instruments.

It cannot, however, be excluded that the systematic differences in the instrument response in different observing modes (timing and imaging) and the systematic uncertainties in the absolute flux calibration (for XMM-Newton it is believed to be around $10 \%^{1}$ ) are responsible for the observed flux differences. We conclude, therefore, that these differences are not sufficient to claim long-term variability of the source. Our results are, therefore, consistent with the CCO hypothesis for XMMUJ173203.3-344518.

\section{Timing analysis}

The observations of the CCO in HESS J1731-347 with Chandra in 2010 (see Table 1), performed in timing mode, were used by Halpern \& Gotthelf (2010a) to search for pulsations down to a period of $10 \mathrm{~ms}$. As mentioned in the introduction, the search did not reveal any pulsations with pulsed fraction larger than $\sim 10 \%$, assuming a sinusoidal pulse shape.

In this work, we analyze the more recent timing observations of the CCO performed with the XMM-Newton observatory

1 http://xmm2.esac.esa. int/docs/documents/

CAL-TN-0052.ps.gz 
D. Klochkov et al.: A non-pulsating neutron star in HESS J1731-347/G353.6-0.7

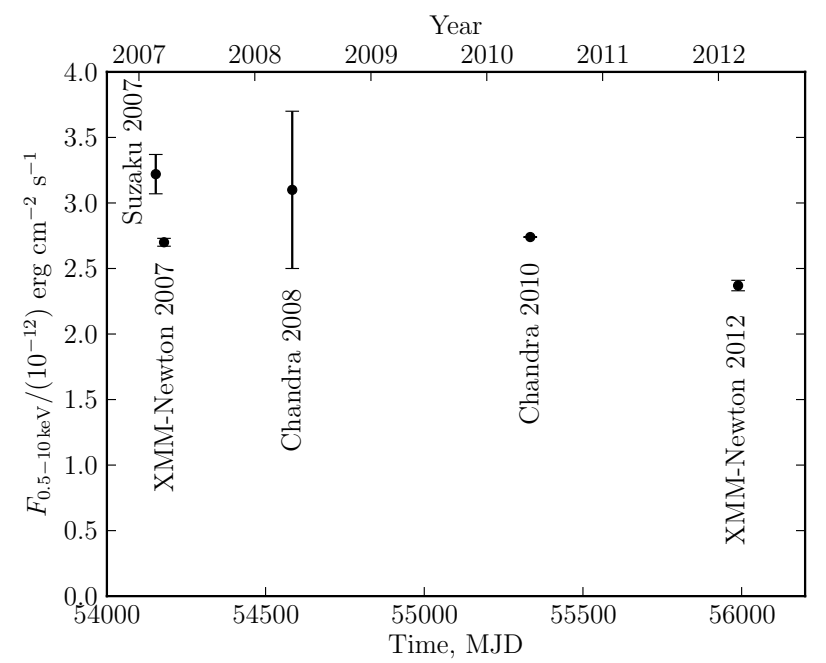

Fig. 1. Absorbed flux measurements of the CCO in HESS J1731-347 in the $0.5-10 \mathrm{keV}$ energy range performed with different instruments. For the data point of Chandra 2010 taken from Halpern \& Gotthelf (2010a), no error bar is available.

(Jansen et al. 2001) in March 2012 with an exposure time of $\sim 23 \mathrm{ks}$. The high time resolution of the EPIC-PN camera in timing mode $(0.03 \mathrm{~ms}$, Strüder et al. 2001) allows the search for coherent pulsations to be extended down to sub-millisecond periods, that is, down to and beyond the theoretical lower limit of the neutron star spin period.

Data processing was performed with the Science Analysis System (SAS) version 12.0.0. No high-background periods had to be excluded from the analysis. We then used a Rayleigh periodogram, also known as $Z_{1}^{2}$-statistics (e.g., Protheroe 1987, and references therein), to calculate the power spectrum down to the period of $0.2 \mathrm{~ms}$ from the event list. The maximum value $Z_{1}^{2}=$ 36.4 is below the $99 \%$ confidence level threshold $Z_{1,99 \%}^{2}=46.3$ and so, no pulsations are detected at this confidence level. The threshold is obtained by calculating the $Z_{1}^{2}$-power for which the probability to exceed it by noise is 0.01 , and taking into account the number of trials (in our case the total number of independent frequencies in the power spectrum, $\sim 1.1 \times 10^{8}$ ). To convert the maximum value of $Z_{1}^{2}$ to an upper limit, we used the approach described by Brazier (1994) and calculated theoretical $Z_{1}^{2}$-distributions for the case of the presence of a signal. The resulting upper limit at $99 \%$ confidence level for the pulsed fraction of a sinusoidal pulsation is $8.3 \%$.

Recently, it has been shown that pulsations of the CCO RX J0822-4300 in the SNR Puppis A exhibit a remarkable phase shift at the energies slightly above $1 \mathrm{keV}$ (Gotthelf \& Halpern 2009). This shift would result in a smearing of pulsations extracted from the data containing photons with energies above and below the energy of the phase shift. The smearing would reduce the sensitivity of our pulsation search technique. To address this point, we performed separate searches for pulsations at the photon energies $E$ above and below $1.5 \mathrm{keV}$ using the procedures described above. In both cases, no pulsation was found. The corresponding $99 \%$ c.l. upper limits on the amplitude of a sinusoidal pulsation are $20.0 \%$ for $E<1.5 \mathrm{keV}$ and $9.4 \%$ for $E>1.5 \mathrm{keV}$.

To conclude on the timing analysis, pulsations of the neutron star from typical $\mathrm{CCO}$ periods of a few seconds down to the fastest possible frequencies can be excluded to below $8.3 \%$ pulsed fraction assuming that no strong energy-dependent shift of the pulse phase is exhibited by the source.

\section{Spectral analysis}

Our spectral study requires a precise measurement of the spectral continuum of the source with a proper treatment/removal of any possible background contribution. Thus, we base our analysis on the $25 \mathrm{ks}$ XMM-Newton data from the observations in 2007. We decided not to include the $S u z a k u$ data because the limited spatial resolution of the XIS cameras does not permit an accurate subtraction of the local diffuse emission generated by the remnant. The 2008 Chandra observations are not used because of the heavy pile-up effect that strongly limits the accuracy of the spectral modeling. The short Swift pointings provide a negligible statistical contribution to the spectra and are therefore also omitted. Finally, we do not use the latest Chandra and XMM-Newton timing observations because of difficulties with finding a proper local source-free region for subtraction of the diffuse emission of the remnant in the two-dimensional slices of the sky available in timing mode observations.

The data processing for all three XMM-Newton instruments (EPIC-PN and two EPIC-MOS, Turner et al. 2001) was performed with SAS version 8.0.0. To extract the source spectrum, we used a circular extraction region with a radius of 50" centered at the CCO and encompassing more than $90 \%$ of the PSF. The background spectrum is extracted from an annulus region around the source with inner and outer radii of $70^{\prime \prime}$ and $110^{\prime \prime}$ respectively. For the spectral extraction, we used the energy range from 0.5 to $9 \mathrm{keV}$. The spectra from all three cameras are found to be consistent and were, therefore, fitted simultaneously in the subsequent analysis.

The extracted spectrum of the CCO can be well described with an absorbed blackbody model with best-fit temperature $k T=0.49(1) \mathrm{keV}$ and absorption column density $n_{\mathrm{H}}=1.50(4) \times$ $10^{22} \mathrm{~cm}^{-2}$. The fit yields $\chi_{\text {red }}^{2} /$ d.o.f. $=1.08 / 409$. The values are consistent with blackbody values derived from the same data set by Halpern \& Gotthelf (2010c), from the Suzaku 2007 data set by Halpern \& Gotthelf (2010c) and Bamba et al. (2012), and from a joint XMM-Newton/Suzaku fit by Tian et al. (2010).

Since the goal of the present work is to fit the spectrum with the physical neutron star atmosphere model described in the following section, we refrained from searching for more complex parametric models which may slightly improve the fit quality.

\section{A carbon atmosphere model for XMMUJ173203.3-344518}

Generally, an isolated neutron star is expected to be covered by a thin (centimeter-scale) atmosphere, which, depending on its chemical composition, substantially modifies the spectrum of the outcoming thermal radiation. So far, no reliable observational constraints on the atmospheric composition of isolated neutron stars have been obtained. Recently, Ho \& Heinke (2009) successfully applied a carbon atmosphere model to the data of the CCO in the Cas A supernova remnant. The analysis allowed them to reconcile the emitting surface area obtained from the spectral fit with the canonical radius of the neutron star. Following Ho \& Heinke (2009), we applied a carbon atmosphere model to our data of the CCO in HESS J1731-347. The lack of pulsations (see Sect. 3) suggests that the emitting area of the source is close to the entire surface of the neutron star, similar to the CCO in Cas A. We, therefore, maintained this assumption in our spectral fits. 


\subsection{The carbon atmosphere model}

In this work, we consider relatively cool model atmospheres with effective temperatures less than $5 \mathrm{MK}$. Therefore, we believe that Compton scattering is not important and include only coherent electron scattering. We also do not take into account the influence of magnetic field. The surface field strength $B$ of the star thus needs to be low enough not to affect the model structures and emergent spectra. It is well known that H-like atoms completely change their structure in an external magnetic field when the energy of an electron on the lowest Landau level $\hbar \omega_{\mathrm{c}} / 2\left(\omega_{\mathrm{c}}\right.$ is the cyclotron frequency) becomes comparable to the ionization energy for the ground state or, equivalently, when $B$ becomes comparable to $B_{\mathrm{cr}}=m_{\mathrm{e}}^{2} e^{3} c Z^{2} / \hbar^{3} \approx 2.4 \times 10^{9} Z^{2} \mathrm{G}$ (see, e.g., Harding \& Lai 2006). For carbon, $B_{\mathrm{cr}} \approx 8.5 \times 10^{10} \mathrm{G}$. We believe that the magnetic field below $(0.001-0.01) \times B_{\mathrm{cr}} \approx 10^{8}-10^{9} \mathrm{G}$ will not noticeably change the ionization energies and number densities of the carbon ions. Thus, we believe that our models are applicable for $B \lesssim 10^{8}-10^{9} \mathrm{G}$. Since no pulsations are detected in XMMUJ173203.3-344518, no estimates of the surface $B$-field strength are available. The results of our modeling are thus valid under the assumption that the surface magnetic field strength of the neutron star is below the specified limit.

The basic assumptions of the computation of stellar atmosphere models with coherent electron scattering are described in Mihalas (1978). Here, we emphasize the differences caused by carbon domination in the chemical composition and high density of the neutron star atmospheres. The models are computed assuming hydrostatic and radiative equilibria in plane-parallel approximation. The main input parameters are the effective temperature $T_{\text {eff }}$, the chemical composition (in this work, we consider a pure carbon atmosphere), and the surface gravity

$g=\frac{G M}{R^{2}}(1+z)$

where $M$ and $R$ are the mass and radius of the neutron star respectively and $G$ is the gravitational constant. The gravitational redshift $z$ on the star surface is related to the neutron star parameters as

$1+z=\left(1-2 G M / c^{2} R\right)^{-1 / 2}$.

In our calculations we assume local thermodynamic equilibrium (LTE); therefore, the number densities of all ionization and excitation states of carbon are calculated using the Boltzmann and Saha equations. We accounted for the pressure ionization effects on carbon populations using the occupation probability formalism (Hummer \& Mihalas 1988), as described by Hubeny et al. (1994). In addition to electron scattering, we took into account the free-free opacity, as well as the bound-free transitions for all carbon ions using opacities from Verner et al. (1996) and Verner \& Yakovlev (1995; see Ibragimov et al. 2003). Line blanketing is taken into account using carbon spectral lines from the CHIANTI atomic database, Version 3.0 (Dere et al. 1997).

For our computations we used the numerical code ATLAS (Kurucz 1970, 1993) modified to deal with high temperatures (Ibragimov et al. 2003; Suleimanov \& Werner 2007; Rauch et al. 2008).

Examples of emergent spectra and temperature structures are shown in Fig. 2. Absorption edges due to the ions CV and CVI are seen in the low-temperature spectra. At higher temperatures, the number density of $\mathrm{CV}$ becomes very small, so that only the CVI edge is seen. It is clearly visible that the edges change the spectral continuum dramatically, so that it strongly deviates from
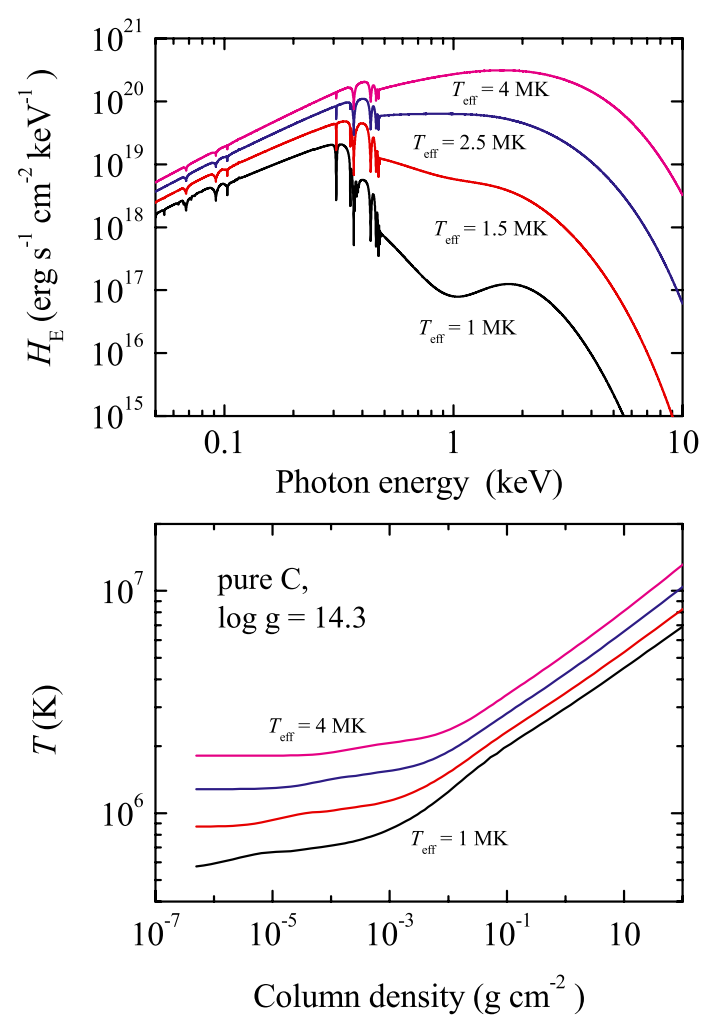

Fig. 2. Emergent spectra (top) and temperature structures (bottom) of pure carbon atmospheres with a fixed $\log g=14.3$ and a set of effective temperatures.

the blackbody spectrum with the corresponding effective temperature or from a spectrum of a hydrogen model atmosphere (see Ho \& Heinke 2009). Our method of the carbon atmosphere modeling will be discussed in detail in a separate paper.

\subsection{Fitting the model to the spectra}

The atmospheres described above are computed for a series of $\log g$ values from 13.70 to 14.90 with a step of 0.15 (the surface gravity $g$ is expressed in cgs units) and effective temperatures $T_{\text {eff }}$ from 1 to $4 \mathrm{MK}$ with a step size of $0.05 \mathrm{MK}$. The photon fluxes have been computed over an energy grid from 0.01 to $20.00 \mathrm{keV}$ with a step size of $0.02 \mathrm{keV}$ sufficiently oversampling the spectral resolution of the instrument. The models are converted into a two-dimensional FITS table that can be imported as an atable model component in the XSPEC or ISIS ${ }^{2}$ spectral fitting packages. Both XSPEC and ISIS allow a linear interpolation between the values in the table, in our case between the effective temperatures and surface gravities, which is found to work very smoothly in our spectral fitting. Additionally, XSPEC and ISIS allow the gravitational redshift $z$ and a normalization factor $K$ to be included to fitting parameters.

In our spectral modeling, we assumed that the carbon atmosphere covers the entire neutron star located at a distance $D$. Taking into account Eqs. (1) and (2), this implies the coupling between the fit parameters

$K=\left(r_{5} / d_{10}\right)^{2}$,

$1+z=\left[1-m\left(0.337 r_{5}\right)^{-1}\right]^{-1 / 2}$,

$\log g=16.125+\log \left[m r_{5}^{-2}(1+z)\right]$, 


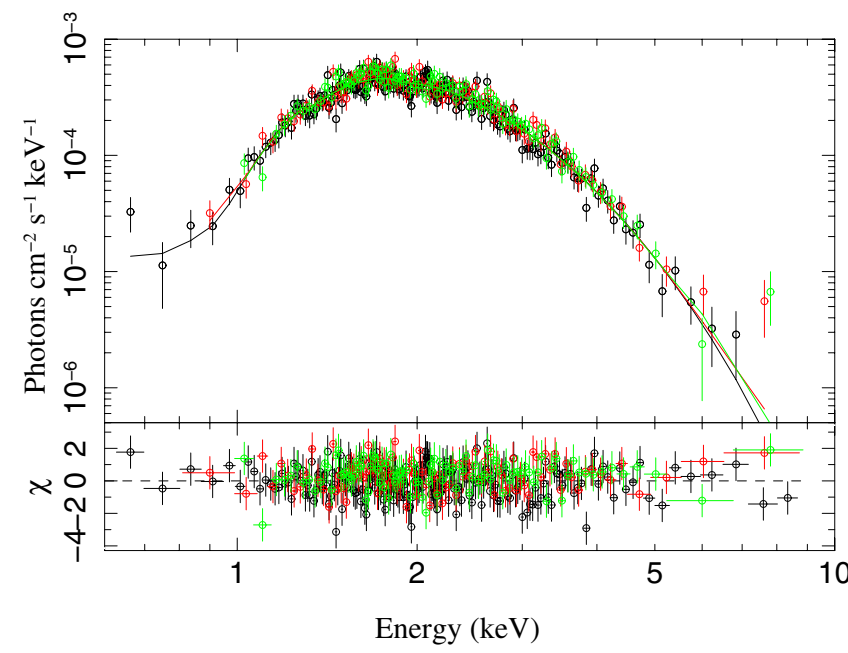

Fig. 3. Fit to the spectra of the CCO in HESS J1731-347 obtained with the PN, MOS1, and MOS2 cameras of XMM-Newton with the carbon atmosphere model assuming a distance of $3.2 \mathrm{kpc}$.

Table 2. Results of the spectral fit of the XMM-Newton data from the CCO in HESS J1731-347 with the carbon atmosphere model described in the text for two fixed distances.

\begin{tabular}{lcc}
\hline \hline Parameter & $D=3.2 \mathrm{kpc}$ & $D=4.5 \mathrm{kpc}$ \\
\hline$n_{\mathrm{H}} /\left(10^{22}\right.$ atoms cm $\left.^{-2}\right)$ & $1.95_{-0.07}^{+0.06}$ & $1.95_{-0.07}^{+0.05}$ \\
$T_{\text {eff }} /\left(10^{6} \mathrm{~K}\right)$ & $2.2_{-0.2}^{+1.2}$ & $2.4_{-0.4}^{+0.9}$ \\
$m$ & $1.5_{-0.6}^{+0.4}$ & $2.2_{-0.9}^{+0.3}$ \\
$r_{5}$ & $12.6_{-5.3}^{+2.1}$ & $15.6_{-5.3}^{+3.6}$ \\
$\chi_{\text {red }}^{2} /$ d.o.f. & $0.98 / 408$ & $0.98 / 408$ \\
$P$-value & 0.59 & 0.59 \\
\hline
\end{tabular}

Notes. The $P$-value is the null hypothesis probability associated to the respective $\chi^{2}$ minimum.

where $K$ is the normalization, $m$ and $r_{5}$ are the mass and the radius of the neutron star in units of solar masses and $10^{5} \mathrm{~cm}$, respectively, and $d_{10}$ is the distance to the star in units of $10 \mathrm{kpc}$. The model is multiplied by a component accounting for interstellar absorption characterized by the equivalent column density of hydrogen atoms $n_{\mathrm{H}}$. As described in the introduction, the source is most probably located either in the Scutum-Crux arm $(\sim 3 \mathrm{kpc})$ or in the Norma-Cygnus arm $(\sim 4.5 \mathrm{kpc})$. The lower limit for the distance derived by Abramowski et al. (2011) on the basis of the X-ray absorption pattern and the absorbing column densities based on CO observations is $\sim 3.2 \mathrm{kpc}$. We thus performed our spectral fitting for the two following fixed distances, 3.2 and $4.5 \mathrm{kpc}$, and assumed the coupling between the parameters specified in Eqs. (3)-(5). For both distances, the model provides a good fit to the data (Fig. 3). The best-fit parameters obtained for the two distances are summarized in Table 2.

\subsection{Constraints to the mass and radius of the neutron star}

Using the carbon atmosphere model, we have explored the $\chi^{2}$-map in the neutron star mass-radius plane. The $\chi^{2}$-contours at 50, 68, and $90 \%$ confidence level for the two fixed distances are shown in Fig. 4. The shaded area in the bottom right (rotation limit) is excluded for neutron stars with a similar equation of state as that of the fastest rotation-powered pulsar known so

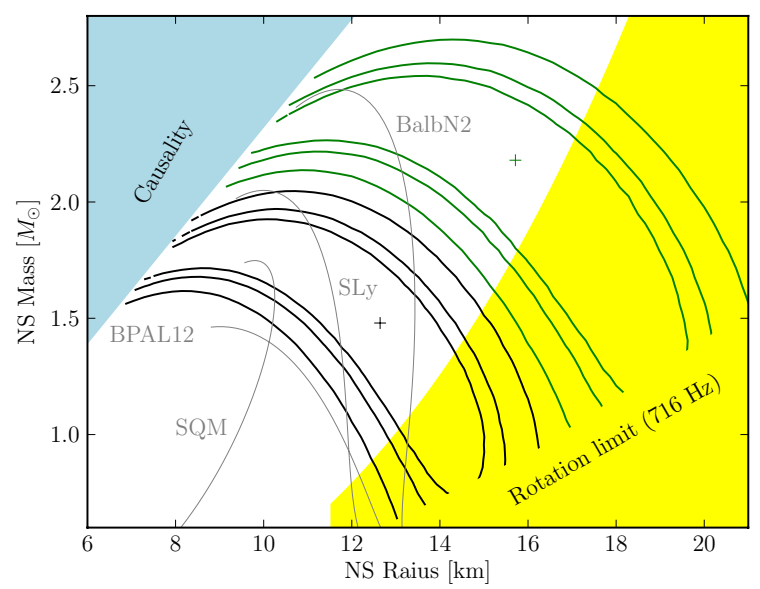

Fig. 4. $\chi^{2}$ confidence regions in the neutron star mass and radius plane for the CCO in HESS J1731-347 obtained with the carbon atmosphere models. The plotted contours correspond to the 50, 68, and $90 \%$ confidence level. The black contours (bottom left) correspond to the fixed distance of $3.2 \mathrm{kpc}$, the green contours (top right) to $4.5 \mathrm{kpc}$. The crosses indicate the corresponding $\chi^{2}$-minima. The shaded areas in the top left and bottom right of the plot indicate the regions excluded by the requirements of causality and from the fastest rotation-powered pulsar known so far, PSR J1748-2446ad (716 Hz).

far, PSR J1748-2446ad (716 Hz). Both $\chi^{2}$-minima are almost equally deep (see Table 2). The fit is, thus, not very sensitive to the distance. Both contours favor somewhat larger mass and radius of the star compared to the canonical values of $1.4 M_{\odot}$ and $10 \mathrm{~km}$, respectively (by a factor of $\sim 20 \%$ or $\sim 50 \%$ for 3.2 and $4.5 \mathrm{kpc}$, respectively). The $3.2 \mathrm{kpc}$ contour is, however, compatible with most nuclear equations of state. We conclude, therefore, that the spectrum of the CCO in HESS J1731-347 is consistent with that of a roughly isotropically emitting neutron star possessing a carbon atmosphere with an effective temperature of $\sim 2.3 \times 10^{6} \mathrm{~K}$ located at a distance of $\sim 3-4 \mathrm{kpc}$. The source is then very similar to the $\mathrm{CCO}$ in the center of the Cas A remnant.

If we fix the neutron star mass to the canonical value of $1.4 M_{\odot}$, the best-fit values of the neutron star radius and the associated $1 \sigma$ uncertainties are $12.7_{-0.9}^{+1.3} \mathrm{~km}$ for the distance of $3.2 \mathrm{kpc}$ and $18.4_{-1.1}^{+1.4} \mathrm{~km}$ for $4.5 \mathrm{kpc}$, respectively. In the case of $4.5 \mathrm{kpc}$, however, the value lies deep beyond the rotation limit set by the fastest rotation-powered pulsar PSR J1748-2446ad (716 Hz; see Fig. 4).

Figure 5 shows the $\chi^{2}$ confidence contours similar to those in Fig. 4 but in the neutron star radius - effective temperature plane. The contours indicate strong degeneracy between the two parameters which is qualitatively well understood. Indeed, a higher neutron star radius corresponds to a larger emitting surface area and, thus, to a higher flux. To reduce the model flux to match the observed value, one needs to reduce the effective temperature, which leads to the observed degeneracy. The gravitational redshift becomes lower with increasing neutron star radius, which compensates for the softening of the spectrum because of the reduction of the effective temperature.

We note that the shape of the contours presented in Figs. 4 and 5 might be affected by a number of effects that are not taken into account in our modeling. These effects are (i) a possible influence of the magnetic field; (ii) the presence of hot spots on the neutron star surface, whose geometry and location, however, prevent the detection of pulsations; and (iii) the actual chemical composition of the stellar atmosphere deviating from pure carbon. 


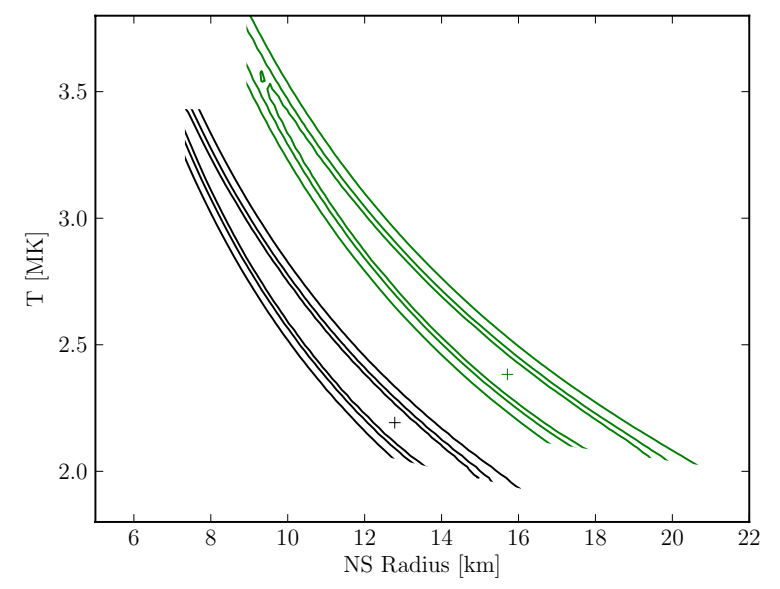

Fig. 5. $\chi^{2}$ confidence regions for the CCO in HESS J1731-347 similar to those in Fig. 4 but in the mass - effective temperature plane. As before, the black contours (bottom left) correspond to the fixed distance of $3.2 \mathrm{kpc}$, the green contours (top right) to $4.5 \mathrm{kpc}$.

\section{Summary and conclusions}

We performed timing and spectral analyses of the X-ray data from the CCO candidate XMMUJ173203.3-344518 located in the center of the shell-type SNR HESS J1731-347 obtained with the XMM-Newton observatory. The data obtained in timing mode allowed X-ray pulsations of the source with an amplitude above $8.3 \%$ to be excluded down to a period of $0.2 \mathrm{~ms}$, that is, well beyond the theoretical lower limit of the neutron star spin period. Based on this finding, we conclude that, unless the spin axis of the neutron star is nearly co-aligned with the observer's line of sight or the emitting regions are located roughly at the star's rotation poles, the observed X-ray emission must be almost uniformly generated by the entire surface of the neutron star.

A fit to the X-ray spectrum of XMMUJ173203.3-344518 with a pure blackbody emission model, assuming the canonical neutron star radius of $10 \mathrm{~km}$, results in an estimated distance to the source of $\sim 30 \mathrm{kpc}$, which is difficult to reconcile with the observed properties of the supernova remnant HESS J1731-347. The distance to the remnant is estimated to be $\sim 3.2-4.5 \mathrm{kpc}$. In this work, we demonstrate that the X-ray spectrum of the CCO candidate can be described with a pure carbon atmosphere model under the assumption that the surface magnetic field of the star does not exceed $\sim 10^{8}-10^{9} \mathrm{G}$. The spectral fit is consistent with a distance to the neutron star of $\sim 3.2-4.5 \mathrm{kpc}$ and a neutron star radius of $\sim 10-15 \mathrm{~km}$. We note that a plausible solution of a similar discrepancy in case of the CCO in Cas A has recently been presented by Ho \& Heinke (2009) who used a carbon atmosphere model to describe the source X-ray spectrum. We thus conclude that, similarly to the CCO in Cas A, XMMUJ173203.3-344518 is most probably a thermally emitting neutron star with an emitting area uniformly distributed over the entire neutron star surface covered by a carbon atmosphere.

Acknowledgements. V.S. acknowledges the support by the German Research Foundation (DFG) grant SFB/Transregio 7 "Gravitational Wave Astronomy" and Russian Foundation for Basic Research (grant 12-02-97006-r-povolzhe-a). We also thank Dr. Craig Heinke for useful comments and suggestions.

\section{References}

Abramowski, A., Acero, F., Aharonian, F., et al. 2011, A\&A, 531, A81 Acero, F., Pühlhofer, G., Klochkov, D., et al. 2009, in Proc. of the 31th ICRC, Lodz [arXiv: 0907.0642]

Aharonian, F. A. 2013, Astropart. Phys., 43, 71

Aharonian, F., Akhperjanian, A. G., Barres de Almeida, U., et al. 2008, A\&A, 477, 353

Bamba, A., Pühlhofer, G., Acero, F., et al. 2012, ApJ, 756, 149

Brazier, K. T. S. 1994, MNRAS, 268, 709

Dere, K. P., Landi, E., Mason, H. E., Monsignori Fossi, B. C., \& Young, P. R. 1997, A\&AS, 125, 149

Gotthelf, E. V., \& Halpern, J. P. 2009, ApJ, 695, L35

Halpern, J. P., \& Gotthelf, E. V. 2010a, ApJ, 725, 1384

Halpern, J. P., \& Gotthelf, E. V. 2010b, ApJ, 709, 436

Halpern, J. P., \& Gotthelf, E. V. 2010c, ApJ, 710, 941

Harding, A. K., \& Lai, D. 2006, Rep. Prog. Phys., 69, 2631

Ho, W. C. G. 2011, MNRAS, 414, 2567

Ho, W. C. G., \& Heinke, C. O. 2009, Nature, 462, 71

Hou, L. G., Han, J. L., \& Shi, W. B. 2009, A\&A, 499, 473

Hubeny, I., Hummer, D. G., \& Lanz, T. 1994, A\&A, 282, 151

Hummer, D. G., \& Mihalas, D. 1988, ApJ, 331, 794

Ibragimov, A. A., Suleimanov, V. F., Vikhlinin, A., \& Sakhibullin, N. A. 2003, Astron. Rep., 47, 186

Jansen, F., Lumb, D., Altieri, B., et al. 2001, A\&A, 365, L1

Kurucz, R. L. 1970, SAO Special Report, 309

Kurucz, R. 1993, ATLAS9 Stellar Atmosphere Programs and $2 \mathrm{~km} \mathrm{~s}^{-1}$ grid. Kurucz CD-ROM No. 13 (Cambridge, Mass.: Smithsonian Astrophysical Observatory), 13

Mihalas, D. 1978, Stellar atmospheres, 2nd edn

Pavlov, G. G., \& Luna, G. J. M. 2009, ApJ, 703, 910

Pavlov, G. G., Sanwal, D., \& Teter, M. A. 2004, in Young Neutron Stars and Their Environments, eds. F. Camilo, \& B. M. Gaensler, IAU Symp., 218, 239 Protheroe, R. J. 1987, PASA, 7, 167

Rauch, T., Suleimanov, V., \& Werner, K. 2008, A\&A, 490, 1127

Reed, J. E., Hester, J. J., Fabian, A. C., \& Winkler, P. F. 1995, ApJ, 440, 706

Strüder, L., Briel, U., Dennerl, K., et al. 2001, A\&A, 365, L18

Suleimanov, V., \& Werner, K. 2007, A\&A, 466, 661

Tian, W. W., Leahy, D. A., Haverkorn, M., \& Jiang, B. 2008, ApJ, 679, L85

Tian, W. W., Li, Z., Leahy, D. A., et al. 2010, ApJ, 712, 790

Turner, M. J. L., Abbey, A., Arnaud, M., et al. 2001, A\&A, 365, L27

Verner, D. A., \& Yakovlev, D. G. 1995, A\&AS, 109, 125

Verner, D. A., Ferland, G. J., Korista, K. T., \& Yakovlev, D. G. 1996, ApJ, 465, 487

Viganò, D., \& Pons, J. A. 2012, MNRAS, 425, 2487 